\title{
Identification of a TMEM127 variant in a patient with paraganglioma and acromegaly
}

\author{
Beryl Stütz'1, Marta Korbonits², Karl Kothbauer³, Werner Müller ${ }^{4}$ and Stefan Fischli1 \\ 1Department of Endocrinology, Diabetes and Clinical Nutrition, Luzerner Kantonsspital, Luzern, Switzerland, \\ 2Department of Endocrinology, Barts and the London School of Medicine, Queen Mary University of London, \\ London, UK, ${ }^{3}$ Department of Neurosurgery, Luzerner Kantonsspital, Luzern, Switzerland, and ${ }^{4}$ Department of \\ Otorhinolarnygology, Head and Neck Surgery, Luzerner Kantonsspital, Luzern, Switzerland
}

Correspondence should be addressed to S Fischli

Email

stefan.fischli@luks.ch

\section{Summary}

The coincidence of a pheochromocytoma or paraganglioma and a pituitary adenoma in the same patient is a rare condition. In the last few years SDHx and MAX mutations have been identified and discussed as a potential causal connection in cases of coincidence. We describe a case of a middle-aged female patient which presented with acromegaly, a growth hormone-secreting pituitary adenoma and a symptomatic neck paraganglioma. The patient was cured by surgery from both the pituitary tumour and the paraganglioma and is well after ten years follow-up. Due to the unusual coexistence of two neuroendocrine tumours, further molecular genetic testing was performed which revealed a variant in the TMEM127 gene (c245-10C>G).

\section{Learning points:}

- Pheochromocytoma/paraganglioma and coexisting functioning pituitary adenoma are a very rare condition. An appropriate treatment of each tumour entity with a multi-disciplinary approach and regular follow-up is needed.

- The possibility of a hereditary disease should be considered and genetic workup is recommended. Genetic testing should focus primarily on the genes with mutations related to pheochromocytomas and paragangliomas.

- Next-generation sequencing with multi-gene panel testing is the currently suggested strategy.

- Genes associated with paragangliomas and pituitary adenomas are SDHA, SDHB, SDHC, SDHD, SDHAF2, MAX and MEN1, while case reports with VHL, RET and NF1 may represent coincidences.

- Variants of uncertain significance may need ongoing vigilance, in case novel data become available of these variants.

\section{Background}

Pheochromocytomas (PCC) and paragangliomas (PGL) are rare neuroendocrine tumours (prevalence between 1:2500 and 1:6667) derived from chromaffin cells of the autonomic nervous system $(1,2)$. In about $30-40 \%$ of all affected patients, germline mutations have been identified in at least 15 susceptibility genes, often without a positive family history (3). Mutations in the $S D H x$ genes (SDHA, SDHB, SDHC, SDHD, SDHAF2 coding for succinate dehydrogenase subunits $\mathrm{A}, \mathrm{B}, \mathrm{C}, \mathrm{D}$, assembly factor 2), VHL (von Hippel-Lindau), RET (MEN2, multiple endocrine neoplasia type 2), NF1 (neurofibromatosis type 1) are the most common, with more rarely identified genes including MAX (MYC-associated factor X), FH (fumarate hydratase) and TMEM127 (transmembrane protein 127 $(2,4,5))$. TMEM127 is located on chromosome $2 \mathrm{q} 11.2$ (familial pheochromocytoma (FP) locus) and was first described in 2005 (6). It encodes a protein with three transmembrane regions, is expressed in normal tissue as well as in different cancer cell lines and acts as a tumour suppressor (7). Until now more than 300 variants of 
the TMEM127 gene are identified by direct sequencing, although in many cases the pathogenicity of these mutations remains uncertain (8). It is expected that TMEM127 mutations are responsible for approximately $2-4 \%$ of PCC and PGL as seen in recent cohort studies (9, $10,11)$. In most cases patients are, similarly to sporadic cases, middle-aged at presentation, but familial cases have also been described (2). While pituitary incidentalomas are relatively common, clinically relevant adenomas are present in 1:1000 of the general population (1). The coexistence of pituitary adenomas and PCC/PGL is extremely rare. The first case of coexisting acromegaly and PCC was described in 1952 (12). Meanwhile the term 'the three P association' ('3PA') has been coined to focus on the association of pituitary adenoma and PCC/PGL and recently a possible causality is being discussed $(1,13)$. Up to now less than 100 patients with 3PAs' had been reported and to our knowledge this is the first case of a patient with clinical manifestation of an acromegaly and a paraganglioma with evidence of a TMEM127 variant (c245-10C $>\mathrm{G}$ ), albeit the clinical significance of this variant is uncertain.

\section{Case presentation}

A 61-year-old female patient presented with pressure in the left neck, deepening of the voice and exertional dyspnoea existing for about half a year. At the same time, paroxysmal hypertensive crisis with systolic blood pressure up to $240 \mathrm{mmHg}$ with headache, flushing and tightness in the chest occurred. In addition, the patient complained about an enlargement of the tongue, ring and shoe size in the last 4 years with recent onset snoring. Except a multinodular goitre, the medical and family history of the patient were unremarkable.

\section{Investigation}

Palpation identified a slightly painful, movable lump in the left supraclavicular fossa, measuring $4 \times 4 \times 6 \mathrm{~cm}$ at MR examination (Fig. 1A and B) leading to relocation of the common carotid artery and inner jugulary vein. The mass was inhomogeneous, partially necrotic and showed clear contrast enhancement. There were no suspicious lymph nodes. Flexible fibreoptic laryngoscopy excluded vocal cord dysfunction. Plasma free metanephrines were in the normal range on two occasions. ${ }^{123}$ I-MIBG-scintigraphy and SPECT/CT demonstrated enhancement of the tumour and possibility of PGL was confirmed (Fig. 2). Insulinlike growth factor 1 (IGF1) and human growth hormone
(GH) were markedly elevated (IGF1 $134 \mathrm{nmol} / \mathrm{L}$ (reference range 9.4-26.9 nmol/L), GH $31.5 \mathrm{mU} / \mathrm{L}$ (reference range $<24 \mathrm{mU} / \mathrm{L}$ )) while other pituitary axes were intact. Suppression of GH by a $75-\mathrm{g}$ oral glucose tolerance test failed and the diagnosis of acromegaly was established. MRI scan showed a $13 \mathrm{~mm}$ pituitary macroadenoma on the left side (Fig. 3A and B).

\section{Treatment}

PGL was treated first by endovascular embolization and then complete surgical resection. Postoperative course
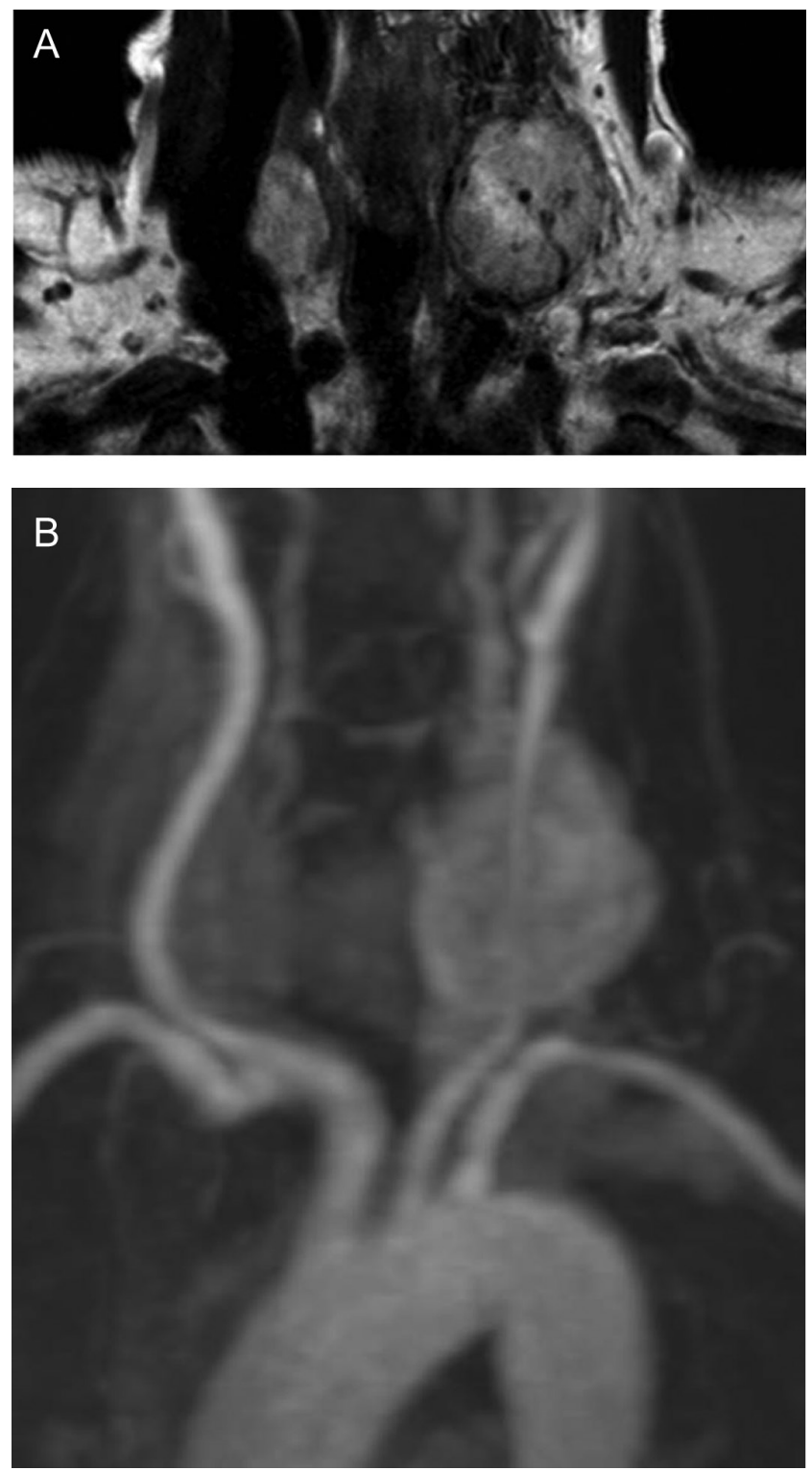

\section{Figure 1}

(A) T2-weighted coronal MRI showing a mass in the left supraclavicular fossa. (B) Coronal MR angiography showing dense vasculature of the tumour. 


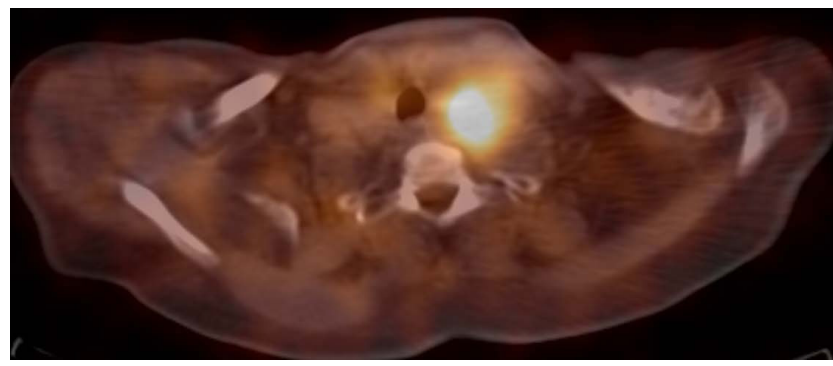

Figure 2

Axial ${ }^{123}$ I-MIBG-scintigraphy/SPECT/CT displaying a hyperintense focus on the left paratracheal side.

was uneventful. Histological workup of the tumour showed synaptophysin and chromogranin positive PGL. Following the PGL surgery, the patient remained with acromegaly symptoms and elevated IGF1. Endoscopic transsphenoidal surgery was performed and confirmed a pituitary adenoma showing densely packed cells with eosinophilic granules in the cytoplasm, positive
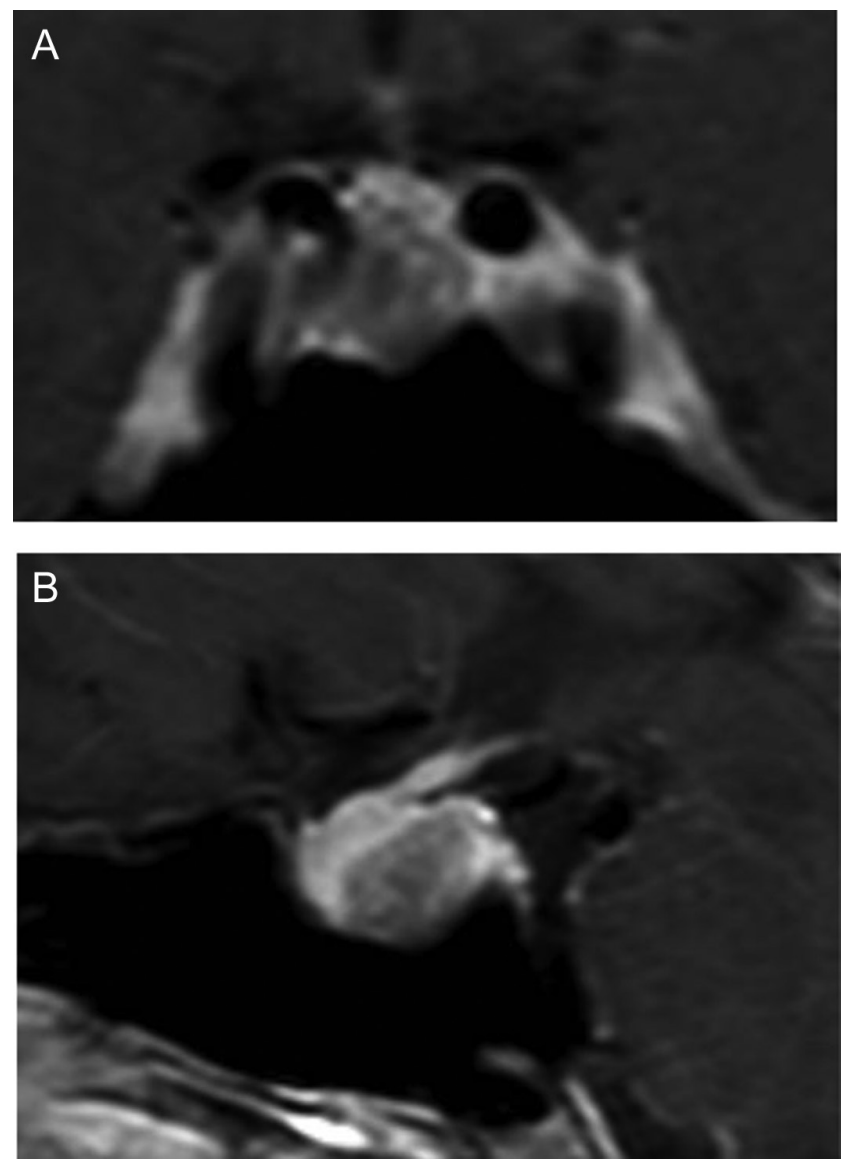

Figure 3

T1-weigthted coronal (A) and sagittal (B) MRI of the sellar region showing a hypointense pituitary macroadenoma. immunohistochemical staining for $\mathrm{GH}$ and a low proliferation index $(\mathrm{Ki}-67<2 \%)$.

\section{Outcome and follow-up}

After the neck and pituitary surgeries, blood pressure remained in the target range and signs of acromegaly, such as macroglossia and snoring reduced. Full suppression on a 75-g oral glucose tolerance test and no residual adenoma on postoperative MRI confirmed remission of the disease. Ultrasound of the neck showed no recurrence of PGL tissue. Colonoscopy showed a few polyps which were partially resected and are under close follow-up. The genetic workup of '3PAs' initially included testing for SDHB, SDHC and SDHD genes, which was negative. A few years later mutation analysis of the FH, MAX, RET, SDHA, SDHAF2, VHL and TMEM127 genes has been added and SDHB, $S D H C$ and SDHD screening were also repeated using next generation sequencing. This resulted in the detection of a heterozygous mutation in the TMEM127 gene on intron 2 (c.245-10C>G), which was a novel variant at that time (in the meantime the same variant was seen in a patient with breast cancer (14), personal communication). Since the patient's parents already deceased determination whether the TMEM127 variant arose de novo was not possible. Also, a screening of the patient's children was declined due to psychiatric disorder. The patient remains cured after ten years of follow-up after pituitary surgery.

\section{Discussion}

While the coexistence of PCC/PGL and pituitary adenoma in the same patient is rare (estimated between 1:2.5 million and 1:8.5 million in the general population (15)), the chance to detect a gene mutation in such a patient is rather high. Possible explanations which are under consideration from O'Toole and colleagues are a common pathogenesis like a PCC/PGL- or a pituitary adenoma-predisposing mutation also leading to the other endocrine tumour, two different mutations or a novel gene mutation causing both tumours, or, less spectacular, an entirely coincidental appearance. Thereby, about one-third of affected patients have either a confirmed genetic mutation in an approved PCC/PGLor pituitary adenoma-predisposing gene or a variant which is potentially pathogenetic and another third has no known mutation but a suggestive personal or family history (1). In 2019, Guerrero-Perez and colleagues reported 10 patients with PCC/PGL (many of them incidentalomas) and coexisting pituitary adenoma. Five 
of them had a GH-secreting pituitary adenoma and five had a PGL, where only one patient had a PGL (located in the neck) in combination with somatotrophinoma. This patient also had a multinodular goiter, similar to our case. They detected a variant of uncertain significance in the RET gene. In the other patients with somatotrophinomas no mutations were found (13). Just as extremely rare as a head and neck PGL (HNPGL) in combination with a GH-secreting pituitary adenoma, TMEM127 mutations are rare causes of a HNPGL. Until now only one mutation carrier with bilateral carotid paraganglioma was reported $(2,10)$. HNPGLs are usually (97-99\%) not associated with catecholamine excess (16), similar to our case where no excess catecholamine secretion was noted. We assume, that the hypertensive episodes described are associated with acromegaly in her case. Another rare condition which should be considered in case of a PCC or PGL in a patient with acromegalic features is the possibility of ectopic GHRH secretion from the PCC/PGL tissue. There are a few case reports, including patients with acromegaly and a sellar mass, but lack of cure after surgical resection, with positive immunostaining for GHRH in a later identified PCC (17). Workup of the pituitary confirmed somatotrophic hyperplasia (histochemical staining for reticulin as a helpful tool) demonstrating also the important impact of an experienced radiologist and pathologist contributing to the right diagnosis. In 2013, Ghazi and colleagues published a compilation of 99 ectopic acromegaly patients emphasizing their own patient with a mediastinal PGL as the GHRH source which represents the only reported ectopic acromegaly secondary to a GHRH secreting PGL (18). In our patient, the fact that the IGF1 elevation persisted after PGL resection and the pituitary tissue showing obvious disruption of the reticulin network, rather than hyperplasia argue against an ectopic GHRH secretion. A genetic workup of Ghazis' patient was not performed and Vieira Neto only excluded a mutation in the RET gene. The identified TMEM127 variant (c.245-10C $>\mathrm{G})$ is a variant of uncertain significance according to ACMG criteria ((19); PM2, as variant is not found in gnomAD database, and BP4, based on in silicio prediction tools). Its location between the other known variants c.245-7 (splice region) and c.245-12 (intron) suggests the possibility of a relevant variant with disruption of the consensus splice site (8). The fact that it is found in a breast cancer patient without TMEM127-related condition does not rule out pathogenicity (14), but whether indeed abnormal splicing occurs has to be confirmed by experimental studies. Disrupted TMEM127 function could lead to increased mTORC1 signalling and thereby induction of cell growth and proliferation, as suggested before (20). The pathogenic impact of TMEM127 mutations were studied in a large cohort of PCC/PGL patients without known mutations in other genes (21). Among them about $2 \%$ had pathogenic mutations in TMEM127 with an estimated penetrance of the disease is over $80 \%$, by the age of 60 years. Therefore, mutation carriers unaffected family members are suggested to have regular MRI follow-up as multiple tumours may develop over time. Interestingly, Bausch et al. also observed other additional malignant neoplasms in 25\% of patients with TMEM127 disease (colon cancer, acute myeloid leukemia, pancreatic adenocarcinoma, malignant melanoma and parathyroid adenoma, but no breast cancer). Against this background, we believe it is key to consider the possibility of hereditary disease when an endocrinologist is confronted with coexisting PCC/PGL and pituitary adenoma ('3PAs') and genetic workup is recommended regarding the available PCC/PGL-related gene panel $(3,5,22,23)$. In our patient the clinical consequence of the described TMEM127 mutation is unknown. The occurrence of the pituitary adenoma and PGL together with the germline mutation can be either pure coincidence or the mutation per se is pathogenic and involved in tumourigenesis. Investigating this question requires additional functional diagnostic testing (i.e. detection of loss of heterozygosity or immunohistochemical staining) which was not done in this particular case.

\section{Declaration of interest}

The authors declare that there is no conflict of interest that could be perceived as prejudicing the impartiality of the research reported.

\section{Funding}

This research did not receive any specific grant from any funding agency in the public, commercial or not-for-profit sector.

\section{Patient consent}

Written informed consent has been obtained from the patient.

\section{Author contribution statement}

BS, MK and SF wrote and edited the manuscript. KK perfomed pituitary surgery and reviewed/edited the manuscript, WM performed paranganglioma surgery and reviewed/edited the manuscript. 


\section{References}

1 O’Toole SM, Denes J, Robledo M, Stratakis CA \& Korbonits M. 15 years OF paraganglioma: the association of pituitary adenomas and phaeochromocytomas or paragangliomas. Endocrine-Related Cancer 201522 T105-T122. (https://doi.org/10.1530/ERC-15-0241)

2 Guha A, Musil Z, Vicha A, Zelinka T, Pacak K, Astl J \& Chovanec M. A systematic review on the genetic analysis of paragangliomas: primarily focused on head and neck paragangliomas. Neoplasma 2019 66 671-680. (https://doi.org/10.4149/neo_2018_181208N933)

3 Eisenhofer G, Klink B, Richter S, Lenders JW \& Robledo M. Metabologenomics of phaeochromocytoma and paraganglioma: an integrated approach for personalised biochemical and genetic testing. Clinical Biochemist: Reviews 201738 69-100.

4 Albattal S, Alswailem M, Moria Y, Al-Hindi H, Dasouki M, Abouelhoda M, Alkhail HA, Alsuhaibani E \& Alzahrani AS. Mutational profile and genotype/phenotype correlation of nonfamilial pheochromocytoma and paraganglioma. Oncotarget 201910 5919-5931. (https://doi.org/10.18632/oncotarget.27194)

5 Sbardella E, Cranston T, Isidori AM, Shine B, Pal A, JafarMohammadi B, Sadler G, Mihai R \& Grossman AB. Routine genetic screening with a multi-gene panel in patients with pheochromocytomas. Endocrine 201859 175-182. (https://doi. org/10.1007/s12020-017-1310-9)

6 Dahia PLM, Hao K, Rogus J, Colin C, Pujana MAG, Ross K, Magoffin D, Aronin N, Cascon A, Hayashida CY, et al. Novel pheochromocytoma susceptibility loci identified by integrative genomics. Cancer Research 200565 9651-9658. (https://doi. org/10.1158/0008-5472.CAN-05-1427)

7 Qin Y, Yao L, King EE, Buddavarapu K, Lenci RE, Chocron ES, Lechleiter JD, Sass M, Aronin N, Schiavi F, et al. Germline mutations in TMEM127 confer susceptibility to pheochromocytoma. Nature Genetics 201042 229-233. (https://doi.org/10.1038/ng.533)

8 Karczewski KJ, Francioli LC, Tiao G, Cummings BB, Alföldi J, Wang Q, Collins RL, Laricchia KM, Ganna A, Birnbaum DP, et al. The mutational constraint spectrum quantified from variation in 141,456 humans. Nature $2020 \mathbf{5 8 1} 434-443$. (https://doi.org/10.1038/s41586020-2308-7)

9 Yao L, Schiavi F, Cascon A, Qin Y, Inglada-Perez L, King EE, Toledo RA, Ercolino T, Rapizzi E, Ricketts CJ, et al. Spectrum and prevalence of FP/TMEM127 gene mutations in pheochromocytomas and paragangliomas. JAMA 2010304 2611-2619. (https://doi. org/10.1001/jama.2010.1830)

10 Neumann HPH, Sullivan M, Winter A, Malinoc A, Hoffmann MM, Boedeker CC, Bertz H, Walz MK, Moeller LC, Schmid KW, et al. Germline mutations of the TMEM127 gene in patients with paraganglioma of head and neck and extraadrenal abdominal sites. Journal of Clinical Endocrinology and Metabolism 201196 E1279-E1282. (https://doi.org/10.1210/jc.2011-0114)

11 Abermil N, Guillaud-Bataille M, Burnichon N, Venisse A, Manivet P, Guignat L, Drui D, Chupin M, Josseaume C, Affres H, et al. TMEM127 screening in a large cohort of patients with pheochromocytoma and/ or paraganglioma. Journal of Clinical Endocrinology and Metabolism 201297 E805-E809. (https://doi.org/10.1210/jc.2011-3360)

12 Iversen K. Acromegaly associated with phaeochromocytoma. Acta Medica Scandinavica 1952142 1-5. (https://doi. org/10.1111/j.0954-6820.1952.tb13837.x)
13 Guerrero-Perez F, Fajardo C, Torres Vela E, Gimenez-Palop O, Lisbona Gil A, Martin T, Gonzalez N, Diez JJ, Iglesias P, Robledo M, et al. 3P association (3PAs): pituitary adenoma and pheochromocytoma/paraganglioma. A heterogeneous clinical syndrome associated with different gene mutations. European Journal of Internal Medicine 201969 14-19. (https://doi. org/10.1016/j.ejim.2019.08.005)

14 National Center for Biotechnology Information. ClinVar; [VCV000639636.1]. (available at: https://www.ncbi.nlm.nih.gov/c linvar/variation/VCV000639636.1). Accessed on 11 June 2020.

15 Denes J, Swords F, Rattenberry E, Stals K, Owens M, Cranston T, Xekouki P, Moran L, Kumar A, Wassif C, et al. Heterogeneous genetic background of the association of pheochromocytoma/paraganglioma and pituitary adenoma: results from a large patient cohort. Journal of Clinical Endocrinology and Metabolism 2015100 E531-E541. (https:// doi.org/10.1210/jc.2014-3399)

16 Offergeld C, Brase C, Yaremchuk S, Mader I, Rischke HC, Gläsker S, Schmid KW, Wiech T, Preuss SF, Suárez C, et al. Head and neck paragangliomas: clinical and molecular genetic classification. Clinics 201267 (Supplement 1) 19-28. (https://doi.org/10.6061/ clinics/2012(sup01)05)

17 Vieira Neto L, Taboada GF, Corrêa LL, Polo J, Nascimento AF, Chimelli L, Rumilla K \& Gadelha MR. Acromegaly secondary to growth hormone-releasing hormone secreted by an incidentally discovered pheochromocytoma. Endocrine Pathology 200718 46-52. (https://doi.org/10.1007/s12022-007-0006-8)

18 Ghazi AA, Amirbaigloo A, Dezfooli AA, Saadat N, Ghazi S, Pourafkari M, Tirgari F, Dhall D, Bannykh S, Melmed S, et al. Ectopic acromegaly due to growth hormone releasing hormone. Endocrine 201343 293-302. (https://doi.org/10.1007/s12020-0129790-0)

19 Richards S, Aziz N, Bale S, Bick D, Das S, Gastier-Foster J, Grody WW, Hegde M, Lyon E, Spector E, et al. Standards and guidelines for the interpretation of sequence variants: a joint consensus recommendation of the American College of Medical Genetics and Genomics and the Association for Molecular Pathology. Genetics in Medicine 201517 405-424. (https://doi. org/10.1038/gim.2015.30)

20 Deng Y, Qin Y, Srikantan S, Luo A, Cheng ZM, Flores SK, Vogel KS, Wang E \& Dahia PLM. The TMEM127 human tumor suppressor is a component of the mTORC1 lysosomal nutrient-sensing complex. Human Molecular Genetics 201827 1794-1808. (https://doi. org/10.1093/hmg/ddy095)

21 Bausch B, Schiavi F, Ni Y, Welander J, Patocs A, Ngeow J, Wellner U, Malinoc A, Taschin E, Barbon G, et al. Clinical characterization of the pheochromocytoma and paraganglioma susceptibility genes SDHA, TMEM127, MAX, and SDHAF2 for gene-informed prevention. JAMA Oncology 20173 1204-1212. (https://doi.org/10.1001/ jamaoncol.2017.0223)

22 Lussey-Lepoutre C, Buffet A, Morin A, Goncalves J \& Favier J. Rodent models of pheochromocytoma, parallels in rodent and human tumorigenesis. Cell and Tissue Research 2018372 379-392. (https:// doi.org/10.1007/s00441-018-2797-y)

23 Gieldon L, William D, Hackmann K, Jahn W, Jahn A, Wagner J, Rump A, Bechmann N, Nölting S, Knösel T, et al. Optimizing genetic workup in pheochromocytoma and paraganglioma by integrating diagnostic and research approaches. Cancers 201911 809. (https:// doi.org/10.3390/cancers11060809) 